\title{
Variation in Readmission Rates by Emergency Departments and Emergency Department Providers Caring for Patients After Discharge
}

\author{
Siddhartha Singh, MD, MS ${ }^{1,2 *}$, Yu-Li Lin, $\mathrm{MS}^{3}$, Ann B. Nattinger, MD, MPH ${ }^{1,2}$, Yong-Fang Kuo, $\mathrm{PhD}^{3}$, James S. Goodwin, $\mathrm{MD}^{3}$
}

${ }^{1}$ Center for Patient Care and Outcomes Research, Medical College of Wisconsin, Milwaukee, Wisconsin; ${ }^{2}$ Division of General Internal Medicine, Department of Medicine, Medical College of Wisconsin, Milwaukee, Wisconsin; ${ }^{3}$ Department of Medicine and Sealy Center on Aging, University of Texas Medical Branch, Galveston, Texas.

BACKGROUND: The role of the emergency department (ED) provider and ED facility in readmissions of recently discharged patients who visit the ED has not been studied.

OBJECTIVE: To determine the variation in readmission rates by ED facility and ED providers caring for patients after discharge.

DESIGN: Retrospective cohort study using multilevel, multivariable models of $100 \%$ Texas Medicare claims data from the years 2007 to 2011 .

SETTING: Texas acute-care hospitals and ED facilities.

PATIENTS: Medicare beneficiaries who visited an ED within 30 days of discharge from a hospital.

INTERVENTION: None.

MEASUREMENT: Readmission after an ED visit within 30 days of discharge from an initial hospitalization defined as a hospitalization starting the day of or the day following the ED visit.

RESULTS: The mean readmission rate following an ED visit was $52.67 \%$. In 2 -level models, $14.2 \%$ of ED providers readmitted significantly more patients (mean readmission rate of $67.2 \%$ ) than the mean; $14.7 \%$ of ED providers readmitted significantly fewer patients (mean readmission rate of $36.8 \%$ ) than the mean. After accounting for the ED facility in 3-level models, the variance for the ED providers decreased $65 \%$ from 0.2532 to 0.0893 .

CONCLUSIONS: The risk of readmission varies by ED provider caring for patients after discharge. A large part of this variation is explained by the ED facility in which the ED providers practice. Thus, ED provider practices patterns and ED facility systems of care may be a target for interventions to reduce readmissions. Journal of Hospital Medicine 2015;10:705-710. (C) 2015 Society of Hospital Medicine
Readmissions of Medicare beneficiaries within 30 days of discharge are frequent and costly. ${ }^{1}$ Concern about readmissions has prompted the Centers for Medicare \& Medicaid Services (CMS) to reduce payments to hospitals with excess readmissions. ${ }^{2}$ Research has identified a number of patient clinical and socio-demographic factors associated with readmissions. ${ }^{3}$ However, interventions designed to reduce readmissions have met with limited success. In a systematic review, no single intervention was regularly effective in reducing readmissions, despite the fact that interventions have targeted both predischarge, transition of care, and postdischarge processes of care. ${ }^{4}$

The different trajectories of care experienced by patients after hospital discharge, and their effect on risk of readmission, have been incompletely studied. Although early outpatient follow-up after discharge is associated with lower readmission rates, ${ }^{5,6}$ a factor

\footnotetext{
*Address for correspondence and reprint requests: Siddhartha Singh, MD, The Medical College of Wisconsin, 9200 West Wisconsin Avenue, Milwaukee, WI, 53226; Telephone: 414-805-0844; Fax: 414-805-0454; E-mail: ssingh@mcw.edu

Additional Supporting Information may be found in the online version of this article.

Received: January 24, 2015; Revised: May 20, 2015; Accepted: May 27, 2015

2015 Society of Hospital Medicine DOI 10.1002/jhm.2407

Published online in Wiley Online Library (Wileyonlinelibrary.com).
}

that has been minimally studied is the role of the emergency department (ED) and the ED provider in readmissions. The ED and ED providers feature prominently in the care received by patients shortly after discharge from a hospital. About a quarter of all hospitalized Medicare patients are evaluated in an ED within 30 days of discharge, ${ }^{7,8}$ and a majority of readmissions within 30 days of discharge are precipitated by an ED visit. ${ }^{9}$ Hence, we asked whether when a recently discharged patient is seen in an ED, does the rate of readmission vary by ED provider and by ED facility?

We used Texas Medicare claims data to examine patients visiting the ED within 30 days of discharge from an initial hospitalization to determine if their risk of readmission varies by the ED provider caring for them and by the ED facility they visit.

\section{METHODS}

\section{Sources of Data}

We used claims from the years 2007 to 2011 for $100 \%$ of Texas Medicare beneficiaries, including Medicare beneficiary summary files, Medicare Provider Analysis and Review (MedPAR) files, Outpatient Standard Analytical Files (OutSAF), and Medicare Carrier files. We obtained diagnosis-related group associated information, including weights, and Major Diagnostic Category from CMS, and used Provider of Services files to determine facility characteristics. 


\section{Establishment of the Study Cohort}

From 2008 through 2011 MedPAR files, we initially selected all hospital discharges from acute-care hospitals in Texas. From these 3,191,160 admissions, we excluded those discharged dead or transferred to other acute-care hospitals $(\mathrm{N}=230,343)$, those who were younger than 66 years at admission $(\mathrm{N}=736,685)$ and those without complete Parts A and B enrollment or with any health maintenance organization enrollment in the 12 months prior to and 2 months after the admission of interest $(\mathrm{N}=596,427)$. From the remaining 1,627,705 discharges, we identified 302,949 discharges that were followed by at least 1 ED visit within 30 days.

We applied the algorithm developed by Kaskie et al. to identify ED visits. ${ }^{10}$ We identified claims for ED services with Current Procedural Terminology (CPT) codes 99281-99285 from Carrier files and bundled claims with overlapping dates or those that were within 1 day of each other. Then we identified claims for ED services using the same CPT codes from OutSAF and bundled those with overlapping dates or those that were within 3 days of each other. Finally, we bundled Carrier and OutSAF claims with overlapping dates and defined them as the same ED visit. From these, we retained only the first ED visit. We excluded those receiving care from multiple ED providers during the $\mathrm{ED}$ visit $(\mathrm{N}=38,565)$, and those who had a readmission before the first ED visit $(\mathrm{N}=1436)$, leaving 262,948 ED visits. For patients who had more than 1 hospitalization followed by an ED visit in a given year, we selected the first hospitalization, resulting in 199,143 ED visits. We then selected ED providers associated with at least $30 \mathrm{ED}$ visits in this cohort, resulting in $1922 \mathrm{ED}$ providers and 174,209 ED visits. For analyses where we examined both ED provider and facility variation in admission rates, we eliminated ED providers that generated charges from more than 1 ED facility, resulting in 525 providers and 48,883 ED visits at $143 \mathrm{ED}$ facilities.

\section{Measures}

\section{Patient Characteristics}

We categorized beneficiaries by age, gender, and ethnicity using Medicare beneficiary summary files. We used the Medicaid indicator as a proxy of low socioeconomic status. We obtained information on weekend admission, emergent admission, discharge destination, and diagnosis-related groupt (DRG) from MedPAR files. We identified comorbidities using the claims from MedPAR, Carrier, and OutSAF files in the year prior to the admission. ${ }^{11}$ We identified total hospitalizations and outpatient visits in the prior year from MedPAR files and Carrier files, respectively. We obtained education status at the level of zip code of residence from the 2011 American Community Survey estimates from the United States Census Bureau. We determined urban or rural residence using the 2013
Rural-Urban Continuum Codes developed by the United States Department of Agriculture.

\section{ED Facility Characteristics}

We used the provider number of the ED facility to link to the Provider of Services files and obtained information on medical school affiliation, facility size, and for profit status.

\section{Study Outcomes}

The outcome of this study was readmission after an ED visit within 30 days of discharge from an initial hospitalization. We defined readmission after an ED visit as a hospitalization starting the day of or the day following the ED visit

\section{Statistical Analyses}

We performed 2-level analyses where patients were clustered with ED providers to examine variation among ED providers. The effect of ED providers was modeled as a random effect to account for the correlation among the patients cared for by the same ED provider. We derived ED provider-specific estimates from models adjusted for patient age, gender, race/ ethnicity, rural or urban residence, Medicaid eligibility, education at the zip code level of residence, and characteristics of the initial admission (emergency admission, weekend admission, discharge destination, its major diagnostic category and DRG weight). We also adjusted for comorbidities, number of hospitalizations, and number of physician visits in the year before the initial admission.

We also conducted 2-level analyses where patients were nested in ED facilities and 3-level analyses where patients were nested in ED providers and ED providers were nested in ED facilities. We adjusted for all factors described above. We computed the change in the variance between 2-level and 2-level analyses to determine the variation in readmission rates that was explained by the ED provider and the ED facility. All analyses were performed with SAS version 9.2 (SAS Institute Inc., Cary, NC).

\section{RESULTS}

We identified 174,209 patients who visited an ED within 30 days of discharge from an initial hospitalization. Table 1 describes the characteristics of these patients as well as the readmission rates associated with these characteristics. The rate of readmission of our cohort of 1,627,705 discharges with or without a following ED visit was $16.2 \%$, whereas the rate of readmission following an ED visit in our final cohort of 174,209 patients was $52.67 \%$. This readmission rate increased with age, from $49.31 \%$ for patients between 66 and 70 years of age to $55.33 \%$ for patients older than 85 years. There were minor variations by gender and ethnicity. Patients residing in metropolitan areas or in zip codes with low education 
TABLE 1. The Effect of Patient Characteristics on the Risk of Hospitalization During an ED Visit Within 30 Days of Hospital Discharge

\begin{tabular}{|c|c|c|c|}
\hline Patient Characteristic & $\begin{array}{l}\text { No. of ED } \\
\text { Visits (\%) }\end{array}$ & \% Readmitted & $\begin{array}{l}\text { Odds Ratio } \\
(95 \% \mathrm{Cl})^{*}\end{array}$ \\
\hline Overall & $174,209(100)$ & 52.67 & \\
\hline \multicolumn{4}{|l|}{ Age, y } \\
\hline $66-70$ & $32,962(18.92)$ & 49.31 & 1.00 \\
\hline $71-75$ & $34,979(20.08)$ & 51.48 & $1.10(1.06-1.13)^{\dagger}$ \\
\hline $76-80$ & $36,728(21.08)$ & 53.01 & $1.15(1.12-1.19)^{\dagger}$ \\
\hline $81-85$ & 34,784 (19.97) & 54.05 & $1.19(1.15-1.23)^{\dagger}$ \\
\hline$>85$ & $34,756(19.95)$ & 55.33 & $1.25(1.21-1.29)^{\dagger}$ \\
\hline \multicolumn{4}{|l|}{ Gender } \\
\hline Male & $71,049(40.78)$ & 52.95 & $1.02(1.00-1.04)$ \\
\hline Female & $103,160(59.22)$ & 52.48 & 1.00 \\
\hline \multicolumn{4}{|l|}{ Race } \\
\hline Non-Hispanic white & $124,312(71.36)$ & 52.77 & 1.00 \\
\hline Black & $16,809(9.65)$ & 51.45 & $0.84(0.81-0.87)^{\dagger}$ \\
\hline Hispanic & 30,618 (17.58) & 52.70 & $0.88(0.85-0.91)^{\dagger}$ \\
\hline Other & $2,470(1.42)$ & 55.71 & $1.06(0.97-1.15)$ \\
\hline \multicolumn{4}{|l|}{ Rural/urban residence } \\
\hline Metropolitan & $136,739(78.49)$ & 53.88 & 1.00 \\
\hline Nonmetropolitan & $35,000(20.09)$ & 48.16 & $0.96(0.93-0.99)^{\dagger}$ \\
\hline Rural & $2,448(1.41)$ & 50.04 & $1.04(0.95-1.13)$ \\
\hline \multicolumn{4}{|l|}{ Medicaid eligible } \\
\hline No & $128,909(74.00)$ & 52.65 & 1.00 \\
\hline Yes & $45,300(26.00)$ & 52.72 & $0.97(0.94-0.99)^{\dagger}$ \\
\hline \multicolumn{4}{|l|}{ Education level $\left.\right|^{\ddagger}$} \\
\hline 1st quartile (lowest) & $43,863(25.18)$ & 54.61 & 1.00 \\
\hline 2nd quartile & $43,316(24.86)$ & 53.92 & $1.00(0.97-1.03)$ \\
\hline 3rd quartile & $43,571(25.01)$ & 50.72 & $0.99(0.96-1.02)$ \\
\hline 4th quartile (highest) & $43,318(24.87)$ & 51.98 & $1.01(0.97-1.04)$ \\
\hline \multicolumn{4}{|l|}{ Emergency admission } \\
\hline No & 99,101 (56.89) & 51.15 & 1.00 \\
\hline Yes & 75,108 (43.11) & 54.68 & $1.07(1.05-1.09)^{\dagger}$ \\
\hline \multicolumn{4}{|l|}{ Weekend admission } \\
\hline No & $131,266(75.35)$ & 52.45 & 1.00 \\
\hline Yes & $42,943(24.65)$ & 53.35 & $1.01(0.99-1.04)$ \\
\hline \multicolumn{4}{|l|}{ Discharge destination } \\
\hline Home & $122,542(70.34)$ & 50.90 & 1.00 \\
\hline Inpatient rehabilitation facility & $9,512(5.46)$ & 55.48 & $1.31(1.25-1.37)^{\dagger}$ \\
\hline Skilled nursing facility & $37,248(21.38)$ & 57.25 & $1.29(1.26-1.33)^{\dagger}$ \\
\hline \multirow[t]{2}{*}{ Other } & $4,907(2.82)$ & 56.88 & $1.14(1.07-1.21)^{\dagger}$ \\
\hline & \multicolumn{2}{|c|}{ Mean \pm SD, Median (Q1-Q3) } & $\begin{array}{l}\text { Odds Ratio } \\
\left(95 \% \mathrm{Cl}^{*}\right.\end{array}$ \\
\hline DRG weight (per unit) & & $1.06(1.05-1.07)^{\dagger}$ \\
\hline $\begin{array}{l}\text { Hospitalization in the prior } \\
\text { year (per hospitalization) }\end{array}$ & \multicolumn{2}{|c|}{$1.03 \pm 1.49,0.00(1.00-2.00)$} & $1.04(1.03-1.04)^{\dagger}$ \\
\hline $\begin{array}{l}\text { Physician visits in the prior } \\
\text { year (per } 10 \text { visits) }\end{array}$ & \multicolumn{2}{|c|}{$11.75 \pm 9.80,5.00(10.00-17.00)$} & $0.97(0.96-0.98)^{\dagger}$ \\
\hline
\end{tabular}

NOTE: There were 141 patients with unknown education level and 22 with unknown place (rural/urban) of residence. These were included as a separate category in the analyses but are not shown. Abbreviations: $\mathrm{Cl}$, confidence interval; DGR, diagnosis-related group; ED, emergency department; SD, standard deviation.

*Estimated from 2-level models adjusted for other patient characteristics.

${ }^{\dagger}$ Statistically significant results.

${ }^{\ddagger}$ Percent of persons age $25+$ years with high school education or higher at the zip code of residence.

levels had higher readmission rates, as did those whose original admission was classified as emergency or those who were not discharged home.

Table 1 also presents the odds of readmission adjusted for all other factors in the table and also adjusted for clustering within ED providers in a

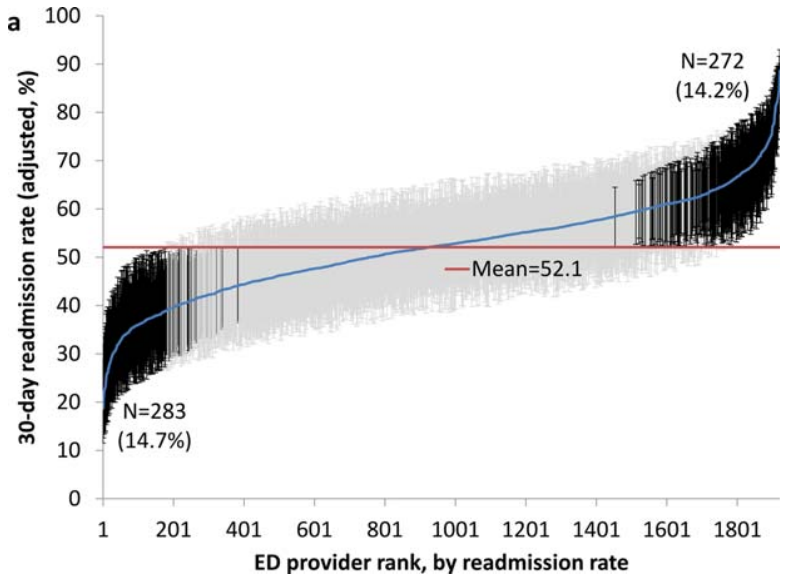

FIG. 1. Ranking of emergency department (ED) provider by adjusted readmission rate: readmission on the day of or day after ED visit. Rates were estimated by 2 -level analyses, adjusted for patient characteristics. The horizontal line represents the overall mean. Error bars represent $95 \%$ confidence intervals of the estimate for the individual ED provider. Black error bars represent ED providers with significantly higher or lower estimates.

2-level model. Increasing age, white race, metropolitan residence, nonhome discharge, higher severity of illness, more hospitalizations in the prior year, fewer physician visits in the prior year, and an emergency initial admission were each associated with a higher readmission rate.

We next generated estimates of readmission rates for each ED provider from the adjusted 2-level models. Figure 1 shows the adjusted cumulative readmission rates for the $1922 \mathrm{ED}$ providers. This figure shows the mean value and $95 \%$ confidence intervals of the readmission rates for each provider. Dark vertical lines indicate providers whose readmission rate differed significantly from the mean adjusted readmission rate of $52.1 \%$ for all providers. Of the ED providers, $14.2 \%$ had significantly higher readmission rates. The mean readmission rate for these 272 providers was $67.2 \%$. Of the ED providers, $14.7 \%$ had significantly lower readmission rates. The mean readmission rate for these 283 providers was $36.8 \%$.

To determine the contribution of the ED facility to the variation in readmission rates, we restricted our analysis to 48,883 patients $(28.06 \%$ of our cohort) seen by 525 ED providers who were associated with only 1 facility (total of 143 facilities). Table 2 describes the unadjusted readmission rates stratified by specific characteristics of those facilities. The unadjusted readmission rate increased with the size of the associated hospital, from $47.61 \%$ for hospitals with less than 100 beds to $57.06 \%$ for hospitals with more than 400 beds. The readmission rate for nonprofit facilities was $53.81 \%$ and for for-profit facilities was $57.39 \%$. Facilities with no medical school affiliation had a readmission rate of $54.51 \%$, whereas those with a major affiliation had a readmission rate of $58.72 \%$. 
TABLE 2. The Effect of ED Facility Characteristics on the Risk of Readmission After an ED Visit

\begin{tabular}{lccl}
\hline ED Facility Characteristic & $\begin{array}{c}\text { No. of ED } \\
\text { Visits (\%) }\end{array}$ & $\begin{array}{c}\% \\
\text { Readmitted }\end{array}$ & $\begin{array}{c}\text { Odds Ratio } \\
(95 \% \text { Cl) }\end{array}$ \\
\hline $\begin{array}{l}\text { Overall } \\
\text { Total beds }\end{array}$ & 48,883 & & \\
$\leq 100$ & $3,936(8.05)$ & 47.61 & 1.00 \\
$101-200$ & $6,251(12.79)$ & 52.07 & $1.38(1.06-1.81)^{\dagger}$ \\
$201-400$ & $13,000(26.59)$ & 56.26 & $1.69(1.32-2.17)^{\dagger}$ \\
$>400$ & $25,696(52.57)$ & 57.06 & $1.77(1.35-2.33)^{\dagger}$ \\
Type of control & $24,999(51.14)$ & 53.81 & 1.00 \\
$\quad$ Nonprofit & $17,108(35.00)$ & 57.39 & $1.32(1.09-1.61)^{\dagger}$ \\
Proprietary & $6,776(13.86)$ & 56.60 & $1.11(0.88-1.41)$ \\
$\quad$ Government & & & \\
Medical school affiliation & $6,487(13.27)$ & 58.72 & 1.00 \\
$\quad$ Major & $7,066(14.45)$ & 56.37 & $0.85(0.58-1.25)$ \\
Limited & $3,164(6.47)$ & 56.19 & $0.71(0.44-1.15)$ \\
Graduate & $32,166(65.80)$ & 54.51 & $0.78(0.57-1.05)$ \\
$\quad$ No affiliation & & & \\
If the same hospital patient & & & \\
$\quad$ was discharged from & $38,532(78.82)$ & 55.64 & $0.96(0.91-1.00)$ \\
Yes & $10,351(21.18)$ & 54.73 & 1.00 \\
No &
\end{tabular}

NOTE: Abbreviations: $\mathrm{Cl}$, confidence interval; $\mathrm{ED}$, emergency department.

"Estimated from 3-level models adjusted for patient characteristics. ED providers associated with only 1 hospital from 2008 through 2011 were selected for the 3-level analyses. There were 525 ED providers from 143 facilities.

†Statistically significant results.

With this smaller cohort, we performed 2 types of 2-level models, where patients clustered within ED facilities and ER providers, respectively, and a 3-level model accounting for clustering of patients within providers and of providers within facilities. From the facility-patient 2-level model, the variance of the ED facility was 0.2718 (95\% confidence interval [CI]: 0.2083-0.3696). From the provider-patient 2-level model, the variance of ED provider was 0.2532 (95\% CI: 0.2166-0.3002). However, when the 3-level model was performed, the variance of ED provider decreased to 0.0893 (95\% CI: $0.0723-0.1132$ ) and the variance of ED facility dropped to 0.2316 (95\% CI: $0.1704-$ $0.3331)$. This indicates $65 \%$ of the variation among ED providers was explained by the ED facility, and in contrast, $15 \%$ of the variation among ED facilities was explained by ED providers.

Table 2 also shows the adjusted odds of readmission generated from the 3-level model. Patients receiving care in ED facilities in hospitals with more beds and in for-profit hospitals were at higher risk for readmission. It is possible that patients seen at the ED associated with the discharging hospital had a lower risk of readmission. This finding was close to being statistically significant $(P=0.051)$.

We repeated all the above analyses using an outcome of readmission anytime between the ED visit and 30 days after discharge from the initial hospitalization (rather than readmission on the day of or after the ED visit). All analyses produced results similar to

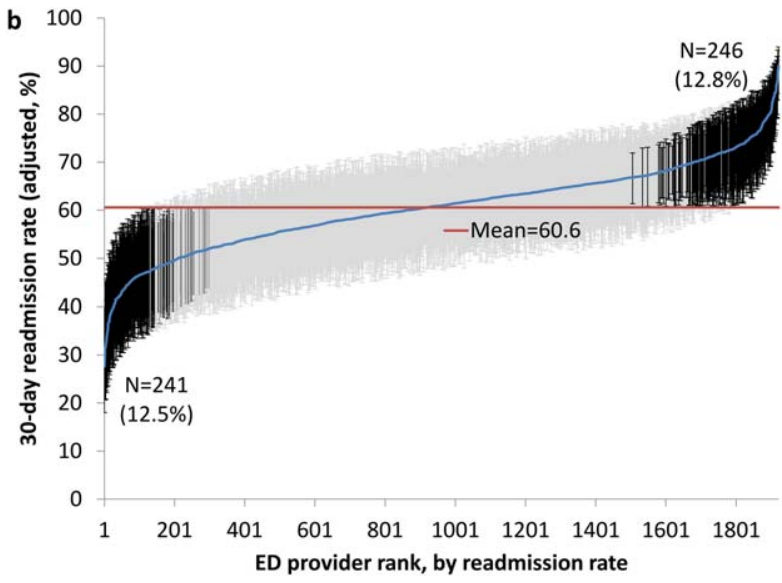

FIG. 2. Ranking of emergency department (ED) provider by adjusted readmission rate: readmission after an ED visit but anytime within 30 days of discharge from initial hospitalization. Rates were estimated by 2-level analyses, adjusted for patient characteristics. The horizontal line represents the overall mean. Error bars represent $95 \%$ confidence intervals of the estimate for the individual ED provider. Black error bars represent ED providers with significantly higher or lower estimates.

the results presented above. For example, Figure 2 shows the adjusted cumulative readmission rates for the 1922 ED providers using this outcome. Of the ED providers, $12.8 \%$ had higher and $12.5 \%$ had lower readmission rates as compared to the mean readmission rate for all ED providers. The Spearman correlation coefficient between the rank of ED providers in immediate readmission rate (Figure 1) and readmission rate within 30 days of hospital discharge (Figure 2) was $0.94(P<0.001)$.

\section{DISCUSSION}

This study found substantial variation in readmission rates by $\mathrm{ED}$ provider, despite controlling for patient clinical and sociodemographic factors. In 3-level models, the ED facility explained a substantial part of the variation by ED provider, with patients seen at larger facilities and for-profit facilities having higher readmission rates.

Variation among ED facilities and ED providers in readmission rates has not previously been studied. There is literature on the variation in ED facility and ED provider admission rates. As readmissions are a subset of all admissions, this literature provides context to our findings. Abualenain et al. examined admission rates for 89 ED physicians for adult patients presenting with an acute medical or surgical complaint at 3 EDs in a health system. ${ }^{12}$ After adjusting for patient and clinical characteristics, admission rates varied from $21 \%$ to $49 \%$ among physicians and from $27 \%$ to $41 \%$ among 3 facilities. Two other studies from single hospitals have found similar variation among providers. ${ }^{13,14}$ The reasons for the variation among ED providers presumably relate to subjective aspects of clinical assessment and the reluctance of providers to rely solely on objective scales, even when 
they are available. ${ }^{14,15}$ Variation in admission rates among different facilities may relate to clustering of providers with similar practice styles within facilities, lack of clinical guidelines for certain conditions, as well as differences among facilities in the socioeconomic status and access to primary care of their clientele. $^{12,16,17}$ For example, Pines et al. have shown that ED facility admission rates are higher in communities with fewer primary care physicians per capita and are influenced by the prevailing county level admission rates. $^{16}$ Capp et al. showed persistent variation in admission rates across hospitals, despite adjusting for clinical criteria such as vital signs, chief complaints, and severity of illness. ${ }^{18}$

Structural differences in ED facilities may also influence the decision to admit. We found that patients visiting ED facilities in hospitals with more beds had a higher readmission rate. ED facility systems of care such as observation units or protocols are associated with lower admission rates. ${ }^{19,20}$ Finally, certain hospitals may actively influence the admission practice patterns of their ED providers. We noted that patients seen at for-profit ED facilities had a greater risk of readmission. A similar finding has been described by Pines et al., who noted higher admission rates at forprofit facilities. ${ }^{16}$ In an extreme example, a recent Justice Department lawsuit alleged that a for-profit hospital chain used software systems and financial incentives to ED providers to increase admissions. ${ }^{21}$

It is possible that the providers with low readmission rates may have inappropriately released patients who truly should have been admitted. A signal that this occurred would be if these patients were readmitted in the days after the ED visits. We examined this possibility by additionally examining readmissions occurring anytime between the ED visit until 30 days after discharge from the initial hospitalization. The results were similar to when we only included readmissions that occurred immediately following the ED visit, with a very high correlation $(r=0.94)$ between the ranking of the ED providers by readmission rates in both circumstances. This suggests that the decisions of the ED providers with low readmission rates to admit or release from the ED were likely appropriate.

Our research has limitations. We studied patients with fee-for-service Medicare in a single large state in the United States over a 4-year period. Our findings may not be generalizable to younger patient populations, other regions with different sociodemographic patterns and healthcare systems, or other time periods. We could not control for many factors that may impact the risk of readmission but are not measured in Medicare databases (eg, clinical data such as vital signs, measures of quality of transition from discharging hospital, ED provider workload). To attribute care to a single ED provider, we excluded patients who were taken care of by multiple ED providers. These patients may have different needs from our study population (eg, more complex issues and longer stays in the ED) and may bias our results.

This study provides a new direction for research and quality improvement targeting readmissions. Research should extend beyond the discharge transition and examine the entire trajectory of posthospitalization care to better understand readmissions. Based directly on this study, research could investigate the practice patterns of ED providers and systems of care at ED facilities that affect readmissions rates. Such investigation could inform quality improvement efforts to standardize care for patients in the ED.

CMS policies hold hospitals accountable for readmissions of the patients they discharge, but do not address the admission process in the ED that leads to readmissions of recently discharged patients. Given the present study, and the fact that the proportion of all hospital admissions that occur through the ED has grown to $44 \%,{ }^{22}$ consideration of the role of the ED in public policy efforts to discourage unnecessary inpatient care may be appropriate.

In summary, this study shows that a recently discharged patient's chances of being readmitted depends partly on the ED provider who evaluates them and on the ED facility at which they seek care. ED provider practice patterns and ED facility systems of care may be a target for interventions aimed at decreasing readmission rates.

Disclosures: This research was supported by grants from the National Institutes of Health (AG033134 and K05CA134923) and from the Agency for Healthcare Research and Quality (R24H5022134). The authors report no conflicts of interest.

\section{References}

1. Jencks SF, Williams MV, Coleman EA. Rehospitalizations among patients in the Medicare Fee-for-Service Program. N Engl J Med. 2009;360:1418-1428.

2. Centers for Medicare \& Medicaid Services. Readmissions reduction program. Available at: http://www.cms.gov/Medicare/Medicare-Feefor-Service-Payment/AcuteInpatientPPS/Readmissions-Reduction-Program.html. Accessed June 192014.

3. Kansagara D, Englander H, Salanitro A, et al. Risk prediction models for hospital readmission: A systematic review. JAMA. 2011;306: $1688-1698$.

4. Hansen LO, Young RS, Hinami K, Leung A, Williams MV. Interventions to reduce 30-day rehospitalization: a systematic review. Ann Intern Med. 2011;155:520-528.

5. Sharma G, Kuo Y, Freeman JL, Zhang DD, Goodwin JS. Outpatient follow-up visit and 30-day emergency department visit and readmission in patients hospitalized for chronic obstructive pulmonary disease. Arch Intern Med. 2010;170:1664-1670.

6. Hernandez AF, Greiner MA, Fonarow GC, et al. Relationship between early physician follow-up and 30-day readmission among Medicare beneficiaries hospitalized for heart failure. JAMA. 2010; 303:1716-1722.

7. Goodman DC, Fisher ES, Chang C. After hospitalization: a Dartmouth Atlas report on post-acute care for Medicare beneficiaries. Dartmouth Atlas website. Available at: www.dartmouthatlas.org/ downloads/reports/Post_discharge_events_092811.pdf. Accessed August 8, 2013.

8. Rising KL, White LF, Fernandez WG, Boutwell AE. Emergency department visits after hospital discharge: a missing part of the equation. Ann Emerg Med. 2013;62:145-150.

9. Kocher KE, Nallamothu BK, Birkmeyer JD, Dimick JB. Emergency department visits after surgery are common for Medicare patients, suggesting opportunities to improve care. Health Aff (Millwood). 2013;32:1600-1607.

10. Kaskie B, Obrizan M, Cook E, et al. Defining emergency department episodes by severity and intensity: a 15-year study of Medicare beneficiaries. BMC Health Serv Res. 2010;10:1-13. 
11. Elixhauser A, Steiner C, Harris DR, Coffey RM. Comorbidity measures for use with administrative data. Med Care. 1998;36:8-27.

12. Abualenain J, Frohna WJ, Shesser R, Ding R, Smith M, Pines JM. Emergency department physician-level and hospital-level variation in admission rates. Ann Emerg Med. 2013;61:638-643.

13. Dean NC, Jones JP, Aronsky D, et al. Hospital admission decision for patients with community-acquired pneumonia: variability among physicians in an emergency department. Ann Emerg Med. 2012;59: 35-41.

14. Mutrie D, Bailey SK, Malik S. Individual emergency physician admission rates: predictably unpredictable. CJEM. 2009;11(2): 149-155.

15. Aujesky D, McCausland JB, Whittle J, Obrosky DS, Yealy DM, Fine MJ. Reasons why emergency department providers do not rely on the pneumonia severity index to determine the initial site of treatment for patients with pneumonia. Clin Infect Dis. 2009;49:e100-e108.

16. Pines JM, Mutter RL, Zocchi MS. Variation in emergency department admission rates across the United States. Med Care Res Rev. 2013;70: 218-231.
17. Venkatesh AK, Dai Y, Ross JS, Schuur JD, Capp R, Krumholz HM. Variation in US hospital emergency department admission rates by clinical condition. Med Care. 2015;53:237-244.

18. Capp R, Ross JS, Fox JP, et al. Hospital variation in risk-standardized hospital admission rates from US EDs among adults. Am J Emerg Med. 2014;32:837-843.

19. Schrock JW, Reznikova S, Weller S. The effect of an observation unit on the rate of ED admission and discharge for pyelonephritis. Am J Emerg Med. 2010;28:682-688.

20. Ross MA, Hockenberry JM, Mutter R, Barrett M, Wheatley M, Pitts SR. Protocol-driven emergency department observation units offer savings, shorter stays, and reduced admissions. Health Aff (Millwood). 2013;32:2149-2156.

21. Creswell J, Abelsonjan R. Hospital chain said to scheme to inflate bills. New York Times. January 23, 2014. Available at: http://www. nytimes.com/2014/01/24/business/hospital-chain-said-to-scheme-to-inflatebills.html?emc=eta1\&_r=0. 23. Accessed April 5, 2014.

22. Schuur JD, Venkatesh AK. The growing role of emergency departments in hospital admissions. N Engl J Med. 2012;367:391-393. 